\title{
Factor Analysis of Motor and Nonmotor Signs in Essential Tremor: Are These Signs All Part of the Same Underlying Pathogenic Process?
}

\author{
Elan D. Louis ${ }^{\mathrm{a}-\mathrm{d}}$ \\ ${ }^{\mathrm{a}}$ GH Sergievsky Center, ${ }^{\mathrm{b}}$ Department of Neurology, and ${ }^{\mathrm{C}}$ Taub Institute for Research on Alzheimer's Disease and \\ the Aging Brain, College of Physicians and Surgeons, Columbia University, and d Department of Epidemiology, \\ Mailman School of Public Health, Columbia University, New York, N.Y., USA
}

\section{Key Words}

Essential tremor $\cdot$ Cerebellar degeneration • Parkinsonism • Dementia

\begin{abstract}
Essential tremor (ET) has traditionally been viewed as monosymptomatic. However, there is an emerging appreciation of an expanded number of motor manifestations as well as a new awareness of nonmotor manifestations. The current goal, through factor analyses, was to determine how these diverse signs relate to one another and shed light on their pathogenic bases. One hundred and thirty-eight ET patients had detailed neurological examinations. In these analyses, three separate factors emerged, explaining $58.7 \%$ of the variance. Factor I was comprised of the hallmark feature of $\mathrm{ET}$, action tremor. It also included intention tremor, which is generally viewed as a sign of cerebellar dysfunction, and tremor duration. Factor II was comprised of cognitive test scores and age, and factor III, of rest tremor. Cognitive test scores did not fall into the same domain as motor features or tremor duration. These results suggest that: (1) the process that underlies cognitive dysfunction in ET is distinct from that which is responsible for action and intention tremors and their progression over time, and (2) cognitive dysfunction in ET is not likely due to cerebellar degeneration. Age loaded with cognitive test scores, further raising the possibility that age-related processes (e.g. Alzheimer-type changes) could underlie cognitive changes in ET.
\end{abstract}

Copyright $\odot 2009$ S. Karger AG, Basel

\section{KARGER}

(c) 2009 S. Karger AG, Basel

Fax +41613061234 E-Mail karger@karger.ch www.karger.com
Accessible online at: www.karger.com/ned

\section{Introduction}

Essential tremor (ET), the most common pathological tremor in humans, is among the more ubiquitous neurological diseases, with a population prevalence of $4.0 \%$ among persons aged 40 and older $[1,2]$. The traditional view of ET, as a monosymptomatic condition characterized only by action tremor, has been supplanted in recent years. The tremor phenomenology is broad and many patients also have other motor manifestations [3]. Thus, intention tremor occurs in the majority of ET cases [4], as do other signs of presumed cerebellar dysfunction (e.g. deficits in tandem gait) [5]. The presence of rest tremor, a parkinsonian feature, noted in a sizable number of ET patients, has also gained increased recognition [6]. In light of recent postmortem findings, in which two patterns of pathology have been described (degenerative changes in the cerebellum in most cases and brainstem Lewy bodies in the remainder) [7], there is added interest in the potential meaning of cerebellar signs and rest tremor in ET and, more specifically, whether these signs are clinical indicators referable to specific pathological substrata.

Along with this evolving understanding of the motor features of ET, it has become increasingly apparent that the disease is associated with certain nonmotor features. Perhaps foremost among these are problems with cognition [8] and, in some patients, frank dementia [9]. The anatomical and pathological basis for these cognitive def-

Dr. Elan Louis

Unit 198, Neurological Institute

710 West 168th Street

New York, NY 10032 (USA)

Tel. +1 212305 9194, Fax +1 212305 1304, E-Mail EDL2@columbia.edu 
icits is unclear. One leading possibility is cerebellar degeneration while another is frontal lobe pathology [10]. Yet another possibility is Alzheimer's disease [11]. If the basis for these cognitive changes were cerebellar, one would expect their onset and severity to parallel that of other cerebellar features (e.g. cerebellar motor signs).

Factor analysis is a useful method to begin to disentangle the relationships between this expanded number of motor and nonmotor manifestations in ET. Factor analysis typically demonstrates that certain clinical signs group with one another, falling into a small number of discrete domains [12].

Several hundred ET patients were enrolled in a crosssectional clinical-epidemiological study. Each was examined in detail and carefully characterized clinically. Using factor analyses, the following a priori hypotheses about possible relationships between the motor and nonmotor manifestations of ET were tested: (1) based on the observation from postmortem studies that most ET cases have evidence of cerebellar degeneration $[7,13]$, the expectation was that intention tremor, which is a cerebellar sign, will fall into the same domain as the hallmark feature of ET (i.e., action tremor), (2) intention tremor, which is a sign of cerebellar dysfunction, and rest tremor, which may be referable to basal ganglia dysfunction, will not be in the same domain as one another, (3) cognitive performance in ET will fall into the same domain as some of the motor signs of ET; however, it is not clear which motor signs (action tremor, intention tremor or rest tremor), and (4) tremor duration will likely correlate with all motor signs as well as cognitive performance. The overarching goal of these analyses was to identify and chart the relationships in ET between cerebellar signs, parkinsonian signs and especially cognitive features and to use these data to begin to shed light on their possible pathogenic bases.

\section{Methods}

\section{Subjects}

ET patients were enrolled in an ongoing cross-sectional, clinical-epidemiological study at the Neurological Institute of New York, Columbia University Medical Center [14], a tertiary referral center in northern Manhattan, New York. Enrollment began in 2000 and continued through 2008. ET patients came from two sources: (1) patients whose neurologist was on staff at the Institute or (2) patients who were cared for by their local doctor in the tristate region (New York, New Jersey, Connecticut) and, as members of the International Essential Tremor Foundation, had read newsletter advertisements for the study. All patients had received a diagnosis of ET from their treating neurologist. Prior to enroll- ment, patients were screened for dementia using the 10-min Telephone Interview for Cognitive Status (TICS) [15]. This was done to minimize the enrollment of individuals with dementia and, therefore, potentially invalid medical histories. On this basis, 1 $(0.2 \%)$ of 465 screened patients (TICS score $=27$ out of 41$)$ was excluded prior to enrollment. The Columbia University Medical Center Internal Review Board approved of all study procedures and written informed consent was obtained at the time of enrollment.

\section{Evaluation}

Once enrolled, all ET cases were evaluated in person by trained testers who administered demographic and medical questionnaires and a videotaped neurological examination [14]. The 20min videotaped neurological examination included assessments of sustained arm extension (postural tremor with arms held outstretched) and 5 tests of kinetic tremor (pouring, drinking, using a spoon, touching finger to nose, and drawing spirals). Each of these tests was performed separately with the dominant arm and nondominant arm (12 tests total). Tremor at rest was assessed in 2 different positions (first with arms resting in lap and, second, while walking). Tremor may occur in ET cases in limbs that are not fully relaxed. Therefore, the trained tester was specifically instructed to ensure that during the rest positions, the patient's arms appeared to be fully relaxed. In August 2005, an assessment of intention tremor (during the finger-nose-finger maneuver) was also added to the protocol.

Videotaped postural and kinetic limb tremor was rated (E.D.L.) using a scale from 0 (no visible tremor) to 3 (large-amplitude tremor) [14]. This rating resulted in an action (postural and kinetic) tremor score in each arm (ratings of $0-3$ on 6 items, range $0-18$ ). These $0-3$ ratings have been validated against quantitative computerized tremor analysis results (e.g. the correlation $r$ between the $0-3$ rating of dominant arm postural tremor and maximal postural tremor amplitude in the dominant arm during computerized tremor analysis $=0.58, \mathrm{p}=0.003$ ) [16]. Rest tremor in the arms or legs was rated using the motor portion of the Unified Parkinson's Disease Rating Scale [17]. Since all ratings were 0 or 1 , this was recoded in the analyses as absent or present. Intention tremor was defined as present when tremor amplitude increased during visually guided movements towards the target. Position-specific tremor or postural tremor at the end of a movement was not included. Similar to Deuschl et al. [4], intention tremor was rated in the terminal period of the finger-nose-finger test: 0 (no intention tremor); 0.5 (probable intention tremor); 1 (definite intention tremor); 2 (incapacitating intention tremor). In addition to the TICS, participants also had further cognitive testing [Mini Mental State Examination (MMSE), score range = 0-30] after enrollment [18].

\section{Final Sample}

Based on the videotaped examination, the diagnosis of ET was confirmed by Dr. Louis in 376 (81.0\%) of 464 patients using published diagnostic criteria that required: (1) either moderate or greater amplitude kinetic arm tremor during at least 3 of 5 tests or a head tremor, and (2) the absence of dystonia or Parkinson's disease (excluding rest tremor) [19]. Eighty-eight (19.0\%) of 464 patients had other diagnoses (Parkinson's disease $=15$, dystonia $=19$, myoclonus $=1$, psychogenic tremor $=1$, enhanced physiological tremor or drug-induced tremor $=52$ ). 
As noted above, the assessment of intention tremor was added 5 years after study initiation, in August 2005. As a consequence, data on intention tremor were available on the $138 \mathrm{ET}$ cases who were enrolled in August 2005 or thereafter. Because intention tremor was a variable of primary interest, the primary analyses used data from these 138 ET cases. One secondary (confirmatory) analysis was also added that used data on the larger sample of 376 ET cases. Although this secondary analysis did not include intention tremor as a variable of interest, it did include each of the other variables of interest (e.g. action tremor, rest tremor) and made use of a larger sample of 376 cases. The sample of 138 (used in the primary analyses) did not differ from the larger sample of 376 (used in one secondary analysis) in terms of age (66.4 \pm 15.4 vs. $67.4 \pm 15.4$ years, $\mathrm{t}=0.65, \mathrm{p}=0.51)$, gender [79 (57.2\%) male vs. $186(49.5 \%)$ male, $\left.\chi^{2}=2.45, \mathrm{p}=0.12\right)$, or tremor duration $(24.4$ \pm 19.4 vs. $22.7 \pm 18.6$ years, $\mathrm{t}=0.91, \mathrm{p}=0.36$ ).

\section{Analyses}

All analyses were performed in SPSS (version 16). In the primary analyses, 10 items were entered into the factor analysis: (1) action tremor score in the dominant arm (range $=0-18)$, (2) action tremor score in the nondominant arm (0-18), (3) rest tremor while seated (absent or present), (4) rest tremor while walking or standing (absent or present), (5) intention tremor in the dominant arm (range $=0-2)$, (6) intention tremor in the nondominant arm $(0-2),(7)$ TICS score (range $=0-41)$, (8) MMSE score (range = $0-30)$, (9) age (years), and (10) duration of action tremor (years). The ratio of ET patients to items entered in these analyses was 138 : 10 (i.e., 13.8:1). A ratio of $>5: 1$ has been suggested as a guideline for the requisite number of patients to items [16], indicating that this sample size was adequate. Factor analysis of binary items (e.g. rest tremor present or absent) has also been shown to be valid; in fact, the resulting factor loadings for binary items generally are attenuated [20]. A correlation matrix was used to examine the correlations between each of the 10 items. In this table, there were 19 correlations, of which only one was $>0.5$ and one other was 0.6 . Factor analysis was performed using the principal component method, first with orthogonal (varimax) rotation and then using the nonorthogonal (oblique) rotation. The former method was chosen to identify separable and independent clusters while the latter was used to identify a general underlying factor structure. Eigenvalues greater than 1.0 were used to select the factors, as is common practice [16]. The one secondary analysis was similar to the primary analyses, except 8 rather than 10 items were entered into the factor analysis (i.e., the 2 intention tremor items were not included).

\section{Results}

There were 138 ET cases (table 1). Factor analysis was performed first with orthogonal (varimax) rotation (table 2). Three factors emerged, explaining $58.7 \%$ of the variance. Factor I, which explained $32.4 \%$ of the variance, was comprised of 5 items: action tremor score in the dominant arm, action tremor score in the nondominant arm, intention tremor in the dominant arm, and intention
Table 1. Demographic and clinical characteristics of 138 ET cases
Age, years

Tremor duration, years

Male gender

Action tremor score in dominant arm (range $=0-18$ )

Action tremor score in nondominant arm (range $=0-18)$

Intention tremor in dominant arm

No intention tremor

Probable intention tremor

Definite intention tremor

Intention tremor in nondominant arm

No intention tremor

Probable intention tremor

Definite intention tremor

TICS score $($ range $=0-41$ )

MMSE score (range $=0-30$ )

Rest tremor present while seated

Rest tremor present while walking or standing
$66.4 \pm 15.4$

$24.4 \pm 19.4$

$79(57.2)$

$9.3 \pm 3.8$

$9.0 \pm 3.7$

$82(59.4)$

$34(24.6)$

$22(15.9)$

$70(50.7)$

$41(29.7)$

27 (19.6)

$36.2 \pm 1.6$

$28.9 \pm 1.3$

$20(14.5)$

$5(3.6)$
Values are means \pm standard deviations or number of cases with percentages in parentheses.

Table 2. Factor analysis (varimax rotation)

\begin{tabular}{|c|c|c|c|}
\hline & $\begin{array}{l}\text { Factor } \\
\text { I }\end{array}$ & $\begin{array}{l}\text { Factor } \\
\text { II }\end{array}$ & $\begin{array}{l}\text { Factor } \\
\text { III }\end{array}$ \\
\hline Eigenvalue & 3.24 & 1.49 & 1.14 \\
\hline Percentage of the variance & 32.4 & 14.9 & 11.4 \\
\hline Action tremor score in dominant arm & 0.66 & -0.27 & 0.17 \\
\hline Action tremor score in nondominant arm & 0.78 & -0.08 & 0.14 \\
\hline Intention tremor in dominant arm & 0.75 & -0.19 & 0.08 \\
\hline Intention tremor in nondominant arm & 0.81 & -0.02 & 0.17 \\
\hline TICS score & -0.15 & 0.66 & 0.06 \\
\hline MMSE score & 0.11 & 0.78 & -0.16 \\
\hline Rest tremor while seated & 0.40 & -0.14 & 0.52 \\
\hline Rest tremor while walking or standing & 0.09 & 0.09 & 0.85 \\
\hline Age in years & 0.25 & -0.57 & -0.41 \\
\hline Tremor duration & 0.70 & 0.19 & -0.22 \\
\hline
\end{tabular}

Eigenvalues after the first three factors were: $0.83,0.77,0.66$, $0.59,0.54,0.47$, and 0.25 . Figures in bold indicate the items that load with each factor.

tremor in the nondominant arm. Interestingly, tremor duration loaded with these items. Factor II, explaining $14.9 \%$ of the variance, was comprised of the 2 cognitive test scores (TICS and MMSE). Of interest is that age (rather than duration) primarily loaded with these two 
Table 3. Factor analysis (oblique rotation)

\begin{tabular}{|c|c|c|c|}
\hline & $\begin{array}{l}\text { Factor } \\
\text { I }\end{array}$ & $\begin{array}{l}\text { Factor } \\
\text { II }\end{array}$ & $\begin{array}{l}\text { Factor } \\
\text { III }\end{array}$ \\
\hline Eigenvalue & 3.24 & 1.49 & 1.14 \\
\hline Percentage of the variance & 32.4 & 14.9 & 11.4 \\
\hline Action tremor score in dominant arm & 0.69 & -0.32 & -0.18 \\
\hline Action tremor score in nondominant arm & 0.79 & -0.15 & -0.15 \\
\hline Intention tremor in dominant arm & 0.77 & -0.25 & -0.09 \\
\hline Intention tremor in nondominant arm & 0.82 & -0.09 & -0.19 \\
\hline TICS score & -0.20 & 0.67 & -0.08 \\
\hline MMSE score & 0.03 & 0.77 & 0.13 \\
\hline Rest tremor while seated & 0.44 & -0.19 & -0.52 \\
\hline Rest tremor while walking or standing & 0.13 & 0.05 & -0.85 \\
\hline Age in years & 0.27 & -0.57 & 0.42 \\
\hline Tremor duration & 0.67 & 0.14 & 0.19 \\
\hline
\end{tabular}

Eigenvalues after the first three factors were: $0.83,0.77,0.66$, $0.59,0.54,0.47$, and 0.25 . Figures in italics indicate the items that load with each factor.

cognitive test scores. The final factor (III) explained $11.4 \%$ of the variance and was comprised of the 2 rest tremor items. Factor analysis was also performed using nonorthogonal (oblique) rotation, and this yielded similar results (table 3 ).

Additional analyses were also performed. First, the 7 (5.1\%) ET cases who had undergone brain surgery (e.g. deep brain stimulation) were removed; in this analysis, the same 3 factors emerged, explaining $59.5 \%$ of the variance. Second, the sample was restricted to the 87 (63.0\%) ET cases who were not currently taking any medications with antitremor effects. The same 3 factors emerged, explaining $60.6 \%$ of the variance. Finally, a confirmatory analysis was performed that used data on the larger sample of 376 ET cases. This confirmatory analysis included 8 items; it did not include the 2 intention tremor items. In this analysis, the same 3 factors emerged, explaining $62.5 \%$ of the variance. As in the primary analyses, the cognitive test score factor (factor II) was distinct from the action tremor/duration factor (factor I) and the rest tremor factor (factor III).

\section{Discussion}

Factor analysis finds relationships or connections between variables that are maximally correlated with one another and minimally correlated with other variables. That is, it identifies clusters, groupings, or clinical di- mensions. These types of analyses are used not only to study the patterns of relationship among many dependent variables but to discover something about the nature of the underlying variables that affect them, even though those independent variables are not measured directly. In the current analyses, the various motor and nonmotor manifestations of ET were not comprised of a single dimension, but rather, formed 3 separable and identifiable clusters. These clusters offer clues about possible underlying pathogenic mechanisms in this poorly understood yet common disease.

Factor I, which explained most of the variance, was comprised of the hallmark feature of ET, namely, action tremor in each arm. Interestingly, intention tremor, which is generally viewed as a sign of cerebellar dysfunction and underlying cerebellar pathology, fell into the same cluster. This confirms a priori hypothesis 1 and is consistent with the results of recent postmortem studies, which have demonstrated cerebellar degenerative changes, including but not restricted to Purkinje cell loss, in the bulk of ET cases $[7,13,21]$. Tremor duration correlated only with action and intention tremor but not with rest tremor or cognitive performance, further indicating that the observed factor structure was genuine.

Factor II was comprised of the two cognitive tests, MMSE and TICS. Of interest is that the cognitive tests did not fall into the same domain as the hallmark or any of the other motor features of ET. Hence, a priori hypothesis 3 was incorrect. Also, the cognitive tests did not fall in the same domain as tremor duration, further suggesting that the process that is resulting in cognitive dysfunction in ET is not the same as the process that is driving the progression of motor features (including intention tremor) with time. It suggests that the cognitive dysfunction is not likely to be due to cerebellar degeneration. Of additional interest is that age loaded most strongly with factor II. The anatomical and pathological basis for cognitive changes in ET is not clear, yet one possibility is they are related to Alzheimer's disease. Clinical studies indicate that age and age of onset are important predictors of cognitive dysfunction in ET, and that ET patients with dementia overwhelmingly have Alzheimer's disease [9, 11]. A postmortem study found more Alzheimer-type changes in the brains of elderly ET cases than age-matched controls [13]. Furthermore, Alzheimer-type changes in the brain are, in general, strongly associated with advancing age [22]. Increased burden of Alzheimer's disease and Alzheimer-type pathology are found in patients with other degenerative diseases (e.g. Parkinson's disease) [23], and it is entirely possible that a similar co-susceptibility 
to a second neurodegenerative disease occurs in ET as well.

Factor III was comprised of rest tremor. Hence, a priori hypothesis 2 was correct (cerebellar signs and rest tremor were not in the same domain). This finding is consistent with the proposition put forth in postmortem studies that there may be two forms of ET: those brains with cerebellar degeneration and those with Lewy bodies (and possibly some features of parkinsonism). These findings are also consistent with the notion that patients with ET who go on to develop Parkinson's disease may form a different clinical-pathological subgroup than the ET patients who do not develop Parkinson's disease over time.

There are few published data on the factor structure of clinical signs in ET. In one prior study, the factor structure of several of the motor features of ET was examined [16]. The current study differs in important ways from that study [16]. First, the current analyses assess a wider range of motor signs in ET, including cerebellar features (intention tremor). More significant is that the current analyses incorporate for the first time data on nonmotor manifestations (i.e., cognitive features) in ET, the importance of which are gaining increasing recognition. Finally, these analyses also examine the relationships between age, duration and these various motor and nonmotor features of ET. There are no other published factor analyses.

This study has some limitations. First, using the TICS prior to enrollment, patients with very low scores were screened out. However, only $1(0.2 \%)$ of 465 patients was excluded in this manner, indicating that this was not likely to result in selection bias. Second, the approach used in this study was a factor analysis of clinical data. While such data may be used to infer underlying pathogenic mechanisms and serve as a guide for future postmortem studies, this was not a postmortem study of ET $[7,13]$. However, this study also has a number of strengths. First, each patient underwent a standardized assessment that included a detailed, 20-min neurological examination. Clinical features were rated by a senior neurologist specializing in movement disorders (E.D.L.). Second, data on a variety of motor features as well as several measures of cognitive performance were assessed. Finally, monopolizing on these clinical data, statistical methods were employed to uncover clinical patterns of possible pathomechanistic significance.

In summary, these clinical results confirm the heterogeneous nature of ET, a view that is also emerging from recent postmortem studies $[13,21]$. The finding that in- tention tremor, a sign traditionally viewed as cerebellar in nature, loads with the hallmark feature of ET and tremor duration further supports the view of ET as a disorder of progressive cerebellar dysfunction. The distinct loading of cognitive tests as separate from the motor signs, including cerebellar signs, suggests that cognitive dysfunction in ET is not likely due to cerebellar degeneration. The finding that age loaded with these cognitive tests further raises the possibility that age-related processes (e.g. Alzheimer-type changes) could underlie the cognitive changes in ET. Increased burden of Alzheimer's disease and Alzheimer-type pathology are found in patients with other degenerative diseases (e.g. Parkinson's disease) [23], and it is entirely possible that a similar cosusceptibility to a second neurodegenerative disease occurs in ET as well. The pathogenesis of ET and of cognitive impairment in ET are poorly understood; it is hoped that future postmortem studies will use the information generated in this clinical-analytic study to help guide their inquiries.

\section{Acknowledgements}

This study is supported by R01 NS039422 and R01 NS042859 from the National Institutes of Health (Bethesda, Md., USA); the Parkinson's Disease Foundation (New York, N.Y., USA); the Arlene Bronstein Essential Tremor Research Fund (Columbia University), and the Claire O’Neil Essential Tremor Research Fund (Columbia University).

\section{References}

\footnotetext{
1 Benito-Leon J, Bermejo-Pareja F, Morales JM, Vega S, Molina JA: Prevalence of essential tremor in three elderly populations of central Spain. Mov Disord 2003;18:389394.

2 Dogu O, Sevim S, Camdeviren H, Sasmaz T, Bugdayci R, Aral M, Kaleagasi H, Un S, Louis ED: Prevalence of essential tremor: doorto-door neurologic exams in Mersin Province, Turkey. Neurology 2003;61:18041806.

3 Benito-Leon J, Louis ED: Essential tremor: emerging views of a common disorder. Nat Clin Pract Neurol 2006;2:666-678; quiz 2p following 691.

4 Deuschl G, Wenzelburger R, Loffler K, Raethjen J, Stolze H: Essential tremor and cerebellar dysfunction clinical and kinematic analysis of intention tremor. Brain 2000; 123:1568-1580

5 Singer C, Sanchez-Ramos J, Weiner WJ: Gait abnormality in essential tremor. Mov Disord 1994;9:193-196.
} 
6 Cohen O, Pullman S, Jurewicz E, Watner D, Louis ED: Rest tremor in patients with essential tremor: prevalence, clinical correlates, and electrophysiologic characteristics. Arch Neurol 2003;60:405-410.

7 Louis ED, Vonsattel JP: The emerging neuropathology of essential tremor. Mov Disord 2007;23:174-182.

$>8$ Benito-Leon J, Louis ED, Bermejo-Pareja F: Population-based case-control study of cognitive function in essential tremor. Neurology 2006;66:69-74.

$\checkmark 9$ Bermejo-Pareja F, Louis ED, Benito-Leon J: Risk of incident dementia in essential tremor: a population-based study. Mov Disord 2007;22:1573-1580.

-10 Troster AI, Woods SP, Fields JA, Lyons KE, Pahwa R, Higginson CI, Koller WC: Neuropsychological deficits in essential tremor: an expression of cerebello-thalamo-cortical pathophysiology? Eur J Neurol 2002;9:143151.

-11 Benito-Leon J, Louis ED, Bermejo-Pareja F: Elderly-onset essential tremor is associated with dementia. Neurology 2006;66:15001505 .
12 Martinez-Martin P, Garcia Urra D, del Ser Quijano T, Balseiro Gomez J, Gomez Utrero E, Pineiro R, Andres MT: A new clinical tool for gait evaluation in Parkinson's disease. Clin Neuropharmacol 1997;20:183-194.

13 Louis ED, Faust PL, Vonsattel JP, Honig LS, Rajput A, Robinson CA, Pahwa R, Lyons KE, Ross GW, Borden S, Moskowitz CB, Lawton A, Hernandez N: Neuropathological changes in essential tremor: 33 cases compared with 21 controls. Brain 2007;130:3297-3307.

14 Louis ED, Zheng W, Applegate L, Shi L, Factor-Litvak P: Blood harmane concentrations and dietary protein consumption in essential tremor. Neurology 2005;65:391-396.

15 Brandt J, Spencer M, Folstein M: The telephone interview for cognitive status. Neuropsychiatry Neuropsychol Behav Neurol 1988;1:111-117.

16 Louis ED, Applegate L, Factor-Litvak P, Parides $\mathrm{MK}$ : Factor structure of motor signs in essential tremor. Neuroepidemiology 2005; 25:42-47.

17 Fahn S, Elton R, Members of the UPDRS Development Committee: Unified Parkinson's disease rating scale; in Fahn S, Marsden CD Calne DB, Goldtein M (eds): Recent Developments in Parkinson's Disease. Florham Park, Macmillan Health Care Information, 1987, pp 153-163.
18 Folstein MF, Folstein SE, McHugh PR: Minimental state. A practical method for grading the cognitive state of patients for the clinician. J Psychiatr Res 1975;12:189-198.

19 Louis ED, Ford B, Lee H, Andrews H: Does a screening questionnaire for essential tremor agree with the physician's examination? Neurology 1998;50:1351-1357.

20 Shrout PE, Parides M: Conventional factor analysis as an approximation to latent trait models for dichotomous data. Int J Methods Psychiatr Res 1992;2:55-65.

$>21$ Shill HA, Adler CH, Sabbagh MN, Connor DJ, Caviness JN, Hentz JG, Beach TG: Pathologic findings in prospectively ascertained essential tremor subjects. Neurology 2008; 70:1452-1455.

22 Imhof A, Kovari E, von Gunten A, Gold G, Rivara CB, Herrmann FR, Hof PR, Bouras C, Giannakopoulos P: Morphological substrates of cognitive decline in nonagenarians and centenarians: a new paradigm? J Neurol Sci 2007;257:72-79.

23 Caballol N, Marti MJ, Tolosa E: Cognitive dysfunction and dementia in Parkinson disease. Mov Disord 2007;22(suppl 17):S358S366. 